\title{
L'INDICE DES PRIX À LA CONSOMMATION EN FRANCE : ACTEURS ET CONFLITS AUTOUR DE SA MESURE
}

\author{
Florence Jany-Catrice
}

La Découverte | «Revue Française de Socio-Économie »

2019/1 n 22 | pages 19 à 43

ISSN 1966-6608

ISBN 9782348042904

Article disponible en ligne à l'adresse :

https://www.cairn.info/revue-francaise-de-socio-economie-2019-1-page-19.htm

Distribution électronique Cairn.info pour La Découverte.

(C) La Découverte. Tous droits réservés pour tous pays.

La reproduction ou représentation de cet article, notamment par photocopie, n'est autorisée que dans les limites des conditions générales d'utilisation du site ou, le cas échéant, des conditions générales de la licence souscrite par votre établissement. Toute autre reproduction ou représentation, en tout ou partie, sous quelque forme et de quelque manière que ce soit, est interdite sauf accord préalable et écrit de l'éditeur, en dehors des cas prévus par la législation en vigueur en France. Il est précisé que son stockage dans une base de données est également interdit. 


\title{
L'indice des prix à la consommation en France : acteurs et conflits autour de sa mesure
}

\author{
Florence JANY-CATRICE \\ Université Lille, Clersé \\ florence.jany-catrice@univ-lille.fr
}

Cet article propose une analyse socio-économique de l'évolution de la mesure de l'inflation en France. Les diverses discussions externes ou plus feutrées qui ont traversé I'histoire de l'indice tout au long du XXe siècle et dans la première décennie du $\mathrm{XXI}{ }^{\mathrm{e}}$ siècle ont toujours porté sur des questions de répartition et de représentation des richesses. Cependant, les acteurs qui soutiennent ces controverses, de même que le contexte macroéconomique dans lequel ces derniers font valoir leurs arguments, changent : la logique politique mettant en scène des rapports sociaux divers entre le gouvernement, la statistique publique et les syndicats fait progressivement place à des logiques scientifiques, qui donnent aux disputes les plus récentes un caractère a priori plus technique. En conférant une place plus décisive aux études scientifiques, aux statisticiens qui les promeuvent et à un large réseau d'experts transnationaux, ces disputes tiennent davantage à distance les acteurs traditionnels. Les différentes innovations incrémentales distillées dans la mesure de l'inflation permettent alors de la faire, tranquillement, changer de nature.

\section{The consumer price index in France: Recomposition of the actors and conflicts around its measurement}

This article proposes a socio-economic analysis of the evolution of the measure of inflation in France. The different external or more muted discussions over the history of the index - throughout the twentieth century and in the first decade of the twenty-first century - have always dealt with questions of distribution and representations of wealth. However, the actors involved in these disputes have changed, as has the macroeconomic context in which they assert their arguments: the political logic putting forward different social relations between the government, public statistical bodies, and trade unions has made way for more scientific logics, which, a priori, give the most recent disputes a more technical nature. By giving a more decisive role to scientific studies, the statisticians that promote them, and a large network of transnational experts, these disputes keep an increasing distance from traditional actors. The different incremental innovations distilled in the measure of inflation allow for a peaceful change in its nature. 
Mots clés: conventions, indices des prix, inflation, répartition, représentation des richesses, statistiques

Keywords: conventions, price index, inflation, distribution, wealth representations, official statistics

\section{Introduction}

L'indice de l'évolution générale des prix, mesure de l'inflation, fait partie des indicateurs macroéconomiques les plus finement auscultés par les États depuis les premières grandes périodes inflationnistes des années 1950, puis celle des années 1970 lorsque « le type-marchand de monnaie-signe s'est imposé et semble avoir conquis définitivement la science économique (y compris la plus libérale) » [Théret, 2010, p. 140]. Cet indice vient « discipliner » les États dans les années 1980, plus encore à partir de la mise en œuvre en 1992 des critères de Maastricht et durant toute la phase de «qualification» des États à la zone monétaire européenne. L'Indice des prix à la consommation (IPC) devient alors la référence ultime à la stabilisation de la valeur de la monnaie [ibid., p. 140]. En 2017, de nombreux commentateurs de l'économie (experts, médias) s'inquiètent régulièrement de la très faible inflation dans les pays occidentaux, malgré une grande diversité de signaux suggérant qu'« en théorie », elle devrait être de retour (croissance à la hausse, politiques de quantitative easing, etc.).

Très auscultée donc, la mesure de I'IPC soulève des questions théoriques et pratiques souvent « redoutables», qualificatif que l'on retrouve chez différents auteurs [Toutain, 1996; Vanoli, 2002; Coyle, 2014]. Pourtant, la plupart des économistes prennent la donnée statistique de I'IPC comme une donnée [Desrosières, 2008]. Véritable "fiction statistique ", I'IPC a été, il est vrai, progressivement consolidé par des institutions, des pratiques sociales et des dispositifs qui l'ont sédimenté et le font apparaître comme une évidence, tout à la fois comme « contrainte » et « ressource » par ceux qui le mobilisent.

Pourtant, l'indice des prix ne va pas de soi, et cet article propose précisément d'explorer sur longue période la boîte noire de sa mesure dans le cas français. Cette analyse a d'indéniables vertus heuristiques, et plaide pour que les interprétations macroéconomiques endogénéisent davantage les enjeux de mesure. Les diverses disputes externes ou plus feutrées qui traversent l'histoire de l'indice soulignent que les épreuves ont toujours porté et portent toujours (mais à des degrés divers) sur des questions de répartition (relevant du partage de la valeur ajoutée et des inégalités) et de représentation ${ }^{1}$ des « richesses » (comment est compté ce qui « compte»).

${ }^{1}$ Le terme de richesse peut ici prêter à confusion : il ne s'agit pas, dans ce qui suit, de son appellation " comptable » qui envisagerait d'un côté les «richesses » au sens des stocks ou des patrimoines accumulés, et de l'autre, les "productions » au sens des flux. Il s'agit de tenir compte ici des « richesses » dans un sens plus générique qu'empruntent de nombreux mondes sociaux: de manière générale, on "est riche » de ses revenus économiques, mais aussi du 
Une première partie fournit le cadre socio-économique de l'analyse (2.), tandis que les suivantes exposent les principales disputes qui ont marqué la vie de I'IPC sous le double enjeu de répartition/représentation : la politique de l'indice des années 1950 jusqu'au rapport Boskin et ses suites (3.), puis le traité de Maastricht, le passage à l'euro, et le pouvoir croissant d'Eurostat (4.). Cet article montre que les acteurs qui portent ces disputes, de même que le contexte macroéconomique dans lequel ces derniers font valoir leurs arguments, changent : la logique politique mettant en scène des rapports sociaux divers entre le gouvernement, la statistique publique et les syndicats qui a marqué l'histoire de la statistique dès années 1940 aux années 1980 fait progressivement place à des arguments scientifiques, conférant aux discussions les plus récentes un caractère a priori plus technique. Ces arguments sont pourtant euxmêmes enchâssés dans les idées et doctrines dominantes. En conférant une place plus décisive aux études scientifiques, aux statisticiens qui les promeuvent et à un large réseau d'experts transnationaux, des acteurs (syndicats, citoyens) sont tenus à distance. Les différentes innovations incrémentales de la mesure de l'inflation permettent alors de la faire, tranquillement, changer de nature ${ }^{2}$.

\section{Un cadre d'analyse socio-économique de la mesure de l'indice des prix}

L'indice des prix est un dispositif cognitif majeur des économies, que celles-ci soient considérées comme des " économies de marché » ou des «économies monétaires de production». La perspective "socio-économique» développée ici mobilise les outils des sciences sociales propres au champ des sciences studies avec l'idée d'ouvrir « les boîtes noires » de la mesure et des concepts associés [MacKenzie, 2005] et, partant, de dénaturaliser les statistiques macroéconomiques, en montrant que ces concepts sont le résultat d'un «intense travail social » [Orléan, 2004, p. 31]. Cette perspective combine une socio-histoire de la statistique [Porter, 1995 ; Armatte, 2010] et une sociologie de la quantification [Desrosières, 2008]. Elle s'arrime aussi à une économie politique des outils macroéconomiques, dans la veine des travaux de Stapleford [2009].

On place le curseur de l'analyse, dans cet article, sur les dynamiques sociales, les rapports de force et de domination qui « légitiment » certains indicateurs plutôt que d'autres, qui valident certaines techniques au détriment d'autres choix sociotechniques, et qui font advenir certains modèles de représentation. Le propos sera en conséquence centré sur la «fabrique » de l'indice des prix - qui a digéré en amont

\footnotetext{
fait d'être dans un environnement socialement et écologiquement sain, ou encore de la qualité des relations que l'on entretient avec les autres. Ces questions ont été par exemple traitées par Méda [1999] ou encore par la commission Stiglitz et al. [2009].

${ }^{2}$ Cette recherche est fondée sur l'exploration de différents manuels de la statistique (BIT, Eurostat, OCDE, Insee, $B L S)$ et sur le dépouillement d'articles de presse, de rapports du CNIS, de documents produits par les syndicats sur la période 1950-2016 avec l'idée de fournir ainsi un corpus d'énoncés issus d'une pluralité d'univers sociaux. Trente entretiens semi-directifs ont également été réalisés entre janvier 2016 et septembre 2017. D'une durée de 45 minutes à 3 heures, ils ont été majoritairement réalisés avec des économistes-statisticiens de l'Insee, en exercice ou non, et ayant été en lien avec la division des prix. Pour étudier la transformation de cet énoncé qu'est le chiffre de l'inflation, nous avons tenté de suivre "les suites de montage » qui relient les " concepts », des " outils» et des «individus» (ménages enquêtés, statisticiens, pouvoirs publics, BCE, etc.). "Ces montages font apparaître des considérations matérielles, idéologiques, conceptuelles, politiques ou administratives et leur imbrication » [Pénissat, 2009, partie 3, chapitre 8].
} 
diverses théories et tours de main -, sur les conditions historiques, sociales et institutionnelles de son élaboration, et de promotions d'innovations, et sur certains des usages qui en sont faits.

\subsection{L'émergence de l'indice des prix}

Le recours à l'histoire jette les bases des tensions de l'analyse, tantôt complémentaires, tantôt opposées, que l'on retrouve encore à l'œuvre aujourd'hui : une logique de représentation et une logique de répartition. Les auteurs qui étudient l'inflation du point de vue de l'origine de sa mesure ne semblent pas attribuer, les uns et les autres, le même poids à ces deux logiques. L'historienne Touchelay [2014] montre ainsi que l'indice des prix apparaît plutôt avec « la première poussée inflationniste » du début du XXe siècle. Il est censé, dit Touchelay, « apaiser la colère des ménagères qui s'insurgeaient contre la vie chère trois ans plus tôt ». De même, en insistant sur la création, dès 1919, de commissions paritaires départementales du coût de la vie supervisées par une "Commission centrale d'études relatives au coût de la vie » (CCERCV, décret de 1920), I'historien Chélini insiste sur l'importance pour les gouvernements et ménages de la perspective du « coût de la vie » [Chélini, 1998, p. 24]. On retrouve cette même idée de l'indice des prix comme une mesure du coût de la vie (du Reich) en Allemagne dès 1919 [Tooze, 2016³], ainsi qu'en Belgique, Jacques Moden insistant sur les luttes sociales visant l'augmentation du « coût de la vie » [Moden, 1982, p. 7]. Les historiens américains montrent aussi que la colère vis-à-vis de l'augmentation du coût de la vie date du début du XXe siècle. Mais, d'après Stapleford [2009], ce souci ne s'est pas immédiatement accompagné d'un intérêt pour les indicateurs de prix de détail. L'attention était alors majoritairement centrée sur les trusts et les monopoles, les économistes considérant alors que les indices de prix de gros étaient des indicateurs pertinents pour rendre compte de l'activité économique.

De son côté, le statisticien Jean Rouchet [2016] envisage le point de départ de l'élaboration et de l'usage dans la statistique publique d'un indice des prix agrégé lors de la crise financière américaine de 1907. Pour lui, cette crise est à l'origine de la construction d'une estimation de l'activité économique via la mesure des prix. La crise financière de Wall Street de 1907 conduit, selon lui, à une crise dans l'économie réelle, entraînant une forte chute de la production et des importations. Cette crise, d'abord américaine, aurait «ému les pouvoirs publics » français [Rouchet, 2016, p. 5] et conduit au premier observatoire des prix dirigé par Duge de Bernonville. Ces premiers indices de prix auraient été conçus comme des mesures indirectes (des proxy donc) de représentation de l'activité. Par commodité il était plus facile de collecter les évolutions de prix que les évolutions de volumes de production. Les premières estimations empiriques des prix mises en place par la statistique publique dateraient ainsi d'avant les périodes de crises inflationnistes [ibid., p. 5].

${ }^{3}$ "The survey covered foodstuffs, the cost of fuel and rents. The weighting scheme was based on surveys of working-class budgets last taken in 1907. As of 10 December 1919, the local magistrates in all towns with more than 10,000 inhabitants, slightly over 600 in total, were to report prices to the Statistical Office of their Land. To check against manipulation, local committees representing employers and labour countersigned the returns. The Länder Statistical Offices compiled regional aggregates. By early February 1920 the Reich's Statistical Office was in possession, for the first time, of a national figure for the cost of living »[Tooze, 2016, p. 9]. 
Quelle que soit la logique qui ait pu originellement dominer dans le besoin de suivre l'indice des prix, l'idée fondatrice de ce que devait être un indice des prix est énoncée par Lucien March, qui a dirigé la Statistique générale de la France entre 1896 et 1920 : "Un indice de prix, c'est-à-dire un coût d'un genre de vie uniforme s'entend non dans le sens vulgaire, mais dans le sens qui convient pour mesurer d'une façon précise l'effet des changements des prix des choses, indépendamment du changement des habitudes ou des goûts, de l'accroissement ou de la différenciation des besoins » [cité par Rouchet, 2016]. Cette idée est traduite statistiquement par un indice dit de "Laspeyres », c'est-à-dire un indice des prix qui agrège par une moyenne arithmétique tous les biens d'un panier fixe dans le temps, de sorte à capter, en moyenne, l'évolution générale des prix de ce panier. Les prix sont pondérés par le coefficient budgétaire de l'année de base. Le choix initial de l'indice de Laspeyres aux États-Unis comme en France est motivé par deux raisons : celui-ci rend bien compte de l'idée intuitive de March d'une part ; c'est la manière considérée à l'époque comme la moins compliquée et la moins coûteuse de capter l'évolution des prix d'un panier de biens d'autre part ${ }^{4}$.

\subsection{L'évolution du contenu de l'indice des prix}

À cette définition générique du Laspeyres des prix, divers modes de collecte se sont succédé dans le temps (tableau 1). Ces collectes témoignent du fait que la statistique est un "objet-frontière » ou encore un " artefact médiateur » [Desrosières, 2008, tome II, p. 77] qui tente d'établir perpétuellement un lien entre l'idéal-type des définitions et de la théorie, et la « réalité » telle que la statistique voudrait la donner à voir. Elles révèlent également la progression d'un indice des prix visant progressivement l'exhaustivité : l'évolution des modalités de collecte exprime le souci pour les statisticiens de couvrir au fur et à mesure des décennies et des "progrès» de la statistique, les prix de la consommation de tous les ménages et tous les biens et services. Si, entre 1914 et 1949, I'Office national de la statistique français retient en effet un panier de 34 articles et fait le choix de centrer son indice sur la consommation d'une " famille ouvrière de quatre personnes vivant à Paris » comme représentative de ce qui peut apparaître alors comme un «budget type », en 2016 c'est l'ensemble des consommateurs (d'agglomérations de plus de 2000 habitants) et $97 \%$ de la consommation qui sont couverts par la mesure de l'évolution des prix à la consommation (voir tableau 1).

S'il résume les grandes étapes de modifications de l'indice, ce qu'on appelle «les générations » de l'indice, le tableau ci-après peine cependant à entrer dans les interstices des disputes autour de cet objet et présente trop succinctement les innovations procédurales pourtant décisives sur le résultat même de cet indice. Citons-en trois :

- le passage à un indice de Laspeyres chaîné à partir du début des années 1970, qui met à jour chaque année les coefficients budgétaires. L'indice des prix vise ainsi à être au plus près de la consommation des ménages ;

${ }^{4}$ Les autres indices, notamment de Paasche et de Fisher, nécessitent la collecte des quantités consommées pour établir le coefficient budgétaire non seulement l'année de base (cas du Laspeyres), mais aussi les années courantes. 


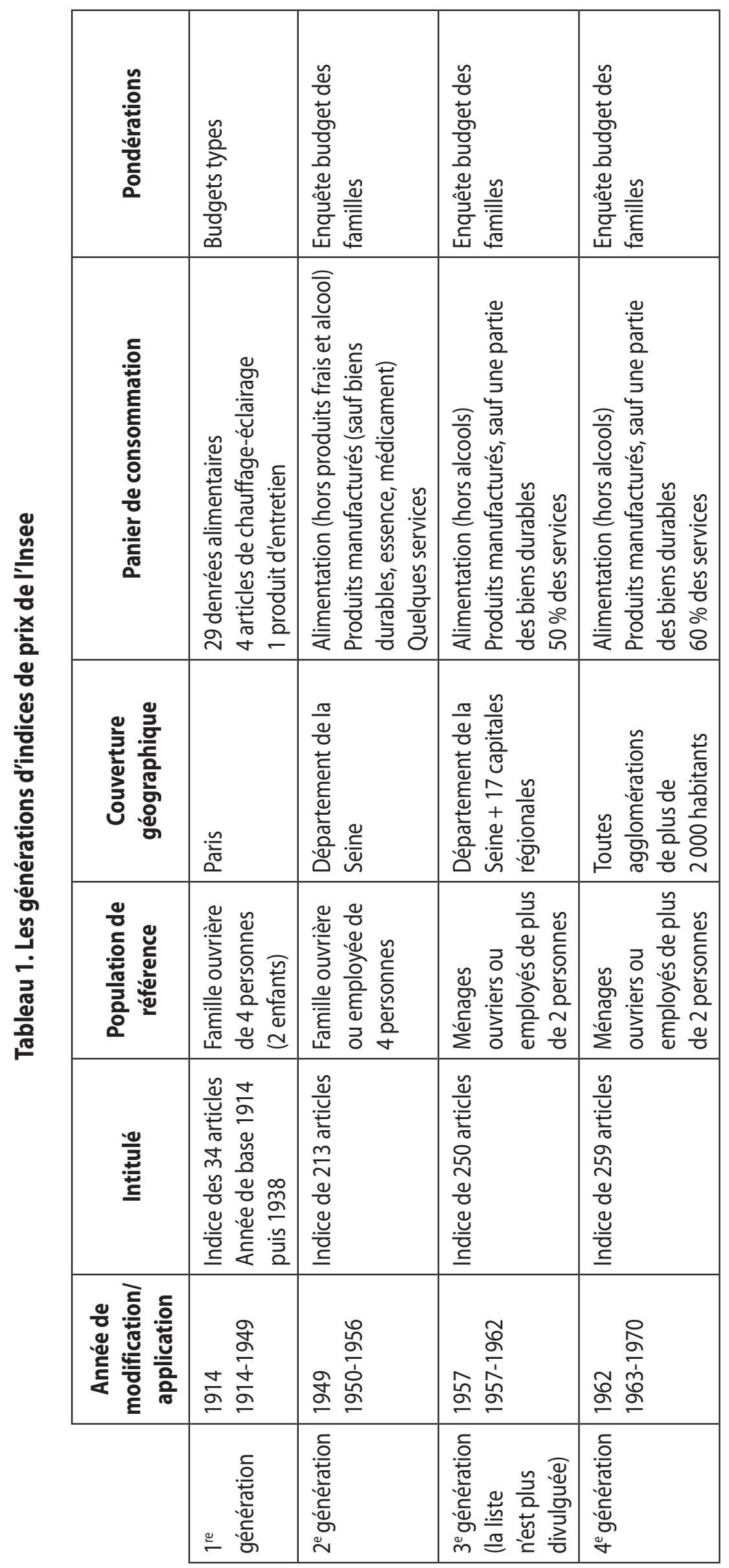

$24 \quad$\begin{tabular}{c|c} 
1er $2019 \mathbf{R f}_{\mathfrak{s e m}}^{22}$ \\
efe
\end{tabular} 


\begin{tabular}{|c|c|c|c|}
\hline 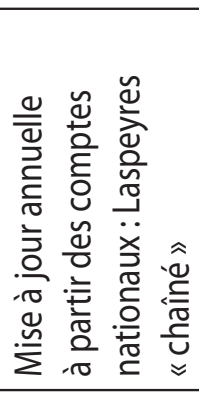 & 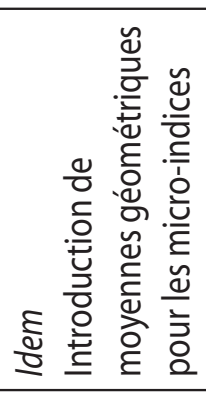 & & \\
\hline 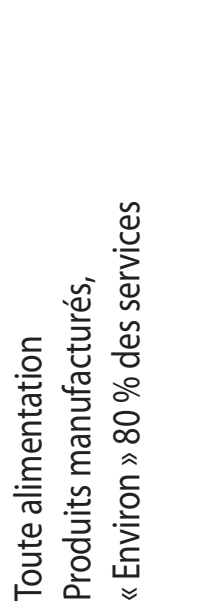 & 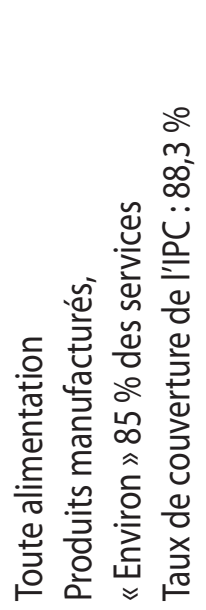 & 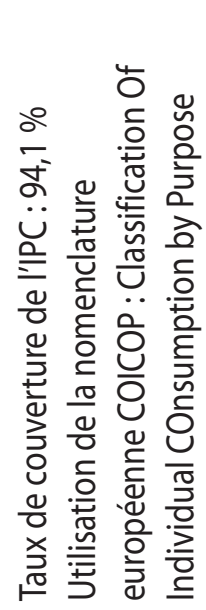 & 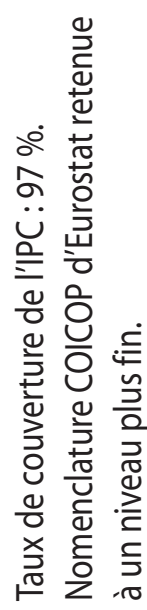 \\
\hline 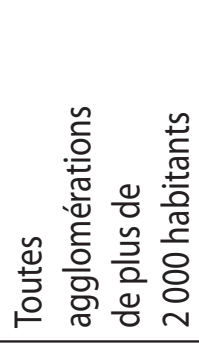 & 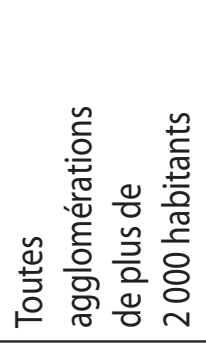 & 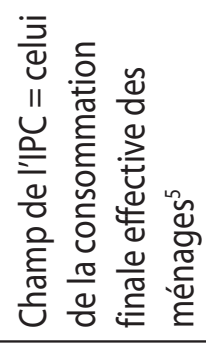 & 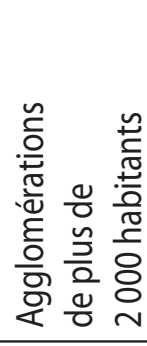 \\
\hline 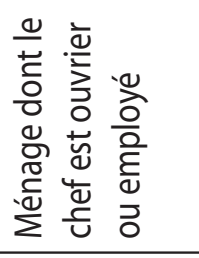 & 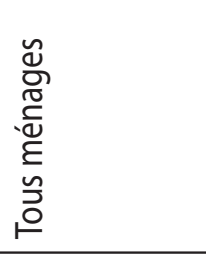 & & \\
\hline 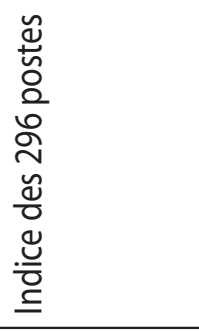 & 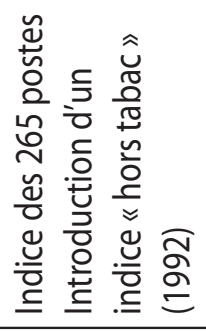 & 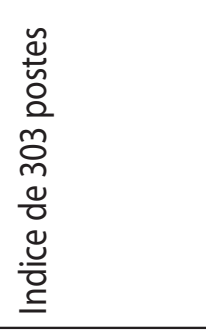 & 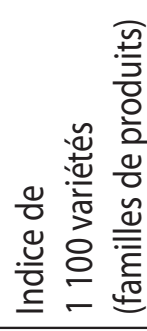 \\
\hline 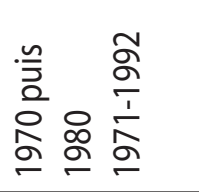 & 용 & ஃু & 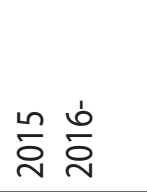 \\
\hline 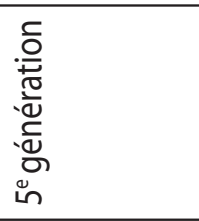 & 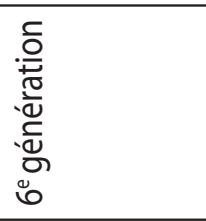 & 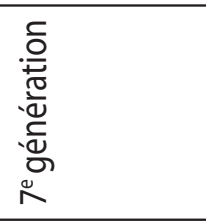 & 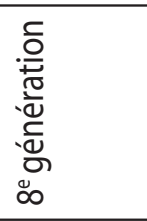 \\
\hline
\end{tabular}


- le recours à des moyennes géométriques (aussi appelées indices de Jevons) pour l'établissement de certains micro-indices de prix de variétés hétérogènes ${ }^{6}$, dès le début des années 1990. Une telle moyenne conduit à réduire quasi mécaniquement l'augmentation des prix;

- un traitement varié de l'effet qualité: méthode phare de ce traitement, la méthode hédonique ${ }^{7}$ a été expérimentée en 1938 aux États-Unis, à partir des travaux de Court, économiste des industriels de l'automobile. L'objectif de Court était de montrer que le prix effectivement subi par le consommateur était moins élevé que l'évolution nominale du prix [Court, 1939]. Durant les années 1980 aux États-Unis, et 1990 en France, la méthode hédonique a été expérimentée, puis appliquée à l'indice pour certains biens d'équipements durables (notamment les ordinateurs). Si Moulton estime en 2001 que $18 \%$ des biens sont traités par la méthode hédonique [Moulton, 2001], celle-ci demeure très circonscrite en France comme aux États-Unis pour plusieurs raisons : I'Insee met en avant le coût élevé du traitement, car le paramétrage économétrique demande toujours plus d'observations pour une efficacité de traitement pas toujours assurée; ensuite, si la méthode est raisonnable pour certains biens semi-durables, elle est inappropriée pour la plupart des biens, et surtout les services consommés qui peinent à être définis par un vecteur de caractéristiques; enfin et surtout, le régime des idées économiques délégitime progressivement les méthodes économétriques et empiriques, au profit de celle de "I'utilité constante » (voir infra). La publication en 2003 d'un article d'Hausman dans Journal of Economic Perspectives apparaît de ce point de vue comme un tournant dans la décrédibilisation de la méthode hédonique au profit d'outils plus conformes à la théorie microéconomique du consommateur.

On revient, dans ce qui suit, sur la " grande transformation» de l'indice des prix à partir de ces «petites innovations » en apparence techniques.

\section{Des disputes des années 1950 aux années post-Boskin}

Le début des années 1950 est marqué par les discussions pour l'élaboration des conventions collectives; elles préparent aussi l'établissement d'un salaire minimum en France.

\subsection{Une première politique de l'indice des prix dans les années 1950}

Les partenaires sociaux sont très engagés dans les débats sur les conventions collectives et élaborent des budgets types, permettant d'estimer quel serait un « panier représentatif » du budget des ménages et de l'évolution de son coût. Ces exercices installent les premiers repères objectivés d'indexation des salaires sur les prix qui

${ }^{6}$ Visant à tenir compte d'effets de substitution: selon cet effet, un consommateur rationnel se tourne régulièrement, pour une même utilité, vers l'achat de biens qui sont devenus relativement moins chers, au détriment d'autres ayant augmenté. L'indice des prix à panier constant ne permet pas de tenir compte de cet effet de substitution; l la moyenne géométrique vise à atténuer en partie cet effet.

${ }^{7}$ Dans une fonction hédonique idéale, toutes les variables qualitatives ou quantitatives qui affectent l'utilité pour le consommateur doivent être prises en compte. 
accompagnent les mécanismes régulateurs du revenu des salariés à partir de la fin des années 1950 [Boyer, 1978, p. 33], à une période où l'État régule les conflits sociaux. On est alors au cœur d'un mouvement d'institutionnalisation de la mesure de l'inflation, « puis d'officialisation de l'usage de cet indice dans les procédures de conciliation et d'arbitrage des salaires» [Boyer, 1978, p. 40]. La loi de 1952 d'indexation du Smig sur la hausse des prix met en place un dispositif appelé «Échelle mobile des salaires ». Ce dispositif consiste à indexer le Smig sur la hausse des prix, si celle-ci est supérieure à $5 \%$ (puis seulement $2 \%$ en 1957). Cela protège le pouvoir d'achat des salariés du risque de l'inflation.

Si elles assurent une forme de stabilité, voire une progression du pouvoir d'achat des salariés, notamment à partir de $1968^{\circ}$, ces premières indexations tendent aussi à déplacer une partie des luttes autour de « la mesure du coût de la vie » - avec tout ce que cette acception contient d'incertain et de normatif. En effet, en modifiant la mesure de l'indice des prix, via l'indexation, c'est le rythme de progression des salaires qui s'en trouve également modifié. C'est dans ce contexte qu'on peut saisir les pressions directes exercées par les différents gouvernements sur les prix des produits " et sur des tarifs publics rentrant dans la composition de l'indice » [Touchelay, 2014, p. 119]. Les enjeux de répartition sont alors au cœur des débats politiques, les pouvoirs publics s'inquiétant des menaces qu'un tel dispositif pouvait entraîner vis-à-vis de l'inflation salariale.

\subsection{Les contestations des années 1960 en France : les syndicats en première ligne}

Alors qu'en France, les années 1950 sont marquées par des rapports de force très soutenus entre État et syndicats autour de la mesure des prix et de l'établissement des budgets types, la CGT marque son insatisfaction dès le début des années 1970 en proposant son propre indice des prix. La crise de confiance majeure vis-à-vis de l'indice qu'incarnera la production de ce « contre-indice » ne peut sans doute pas être déconnectée de ce qui apparaîtra comme une première phase de globalisation financière, lorsque les États-Unis, et le monde occidental à sa suite, abandonnent l'étalonor en 1971, «faisant du capitalisme un monde de monnaies [...] concurrentielles » [Théret, 2010].

En matière d'indice des prix, les premiers acteurs de la contestation sont les syndicats. C'est d'abord sur le terrain de la composition du panier de biens que la CGT s'investit. lls s'interrogent par exemple sur l'inclusion ou non des impôts ou encore sur la part du coefficient budgétaire à allouer aux loyers dans l'indice. La CGT décide également de ne pas tenir compte de l'évolution de la qualité des biens dans le panier fixe qu'elle établit en interne, considérant qu'en tenir compte joue comme une décote sur la mesure de l'évolution des prix, alors même que l'évolution de la qualité est, selon les syndicats, plus contrainte que choisie. "L'indice CGT » est publié tous les mois entre 1973 et 1998, grâce à une collecte réalisée, pour l'essentiel, par des

\footnotetext{
${ }^{8}$ Boyer parle de quasi-indexation de salaires liée à une nouvelle politique du Smig, à l'extension du nombre d'accords signés dans le cadre de conventions collectives comportant des clauses d'échelle mobile, notamment dans les secteurs du textile et de la chimie, et à leur diffusion à d'autres secteurs ensuite [Boyer, 1978, p. 47].
} 
militants et étudiants. Cet indicateur alternatif joue durant les années 1970 et 1980 un rôle social décisif comme véritable levier de négociation. Dans ces années-là, si peu d'entreprises prennent directement cet indicateur alternatif pour négocier les augmentations de salaires, l'indice CGT est hybridé à l'indice officiel, en particulier dans les branches où la CGT est particulièrement bien implantée comme la chimie ou la métallurgie.

La fin de la production de l'indice CGT coïncide avec l'insoutenabilité de la fixité du panier de biens (érigé comme principe par la confédération), tant la période au cours de laquelle l'indice CGT est calculé est marquée par un accroissement de la variété des produits consommés qui rendent difficile le maintien d'un repère hyperfixe. Cette période post-fordiste voit également un changement drastique dans l'orientation des politiques économiques, les politiques monétaristes faisant leur apparition dès la fin des années 1980. C'est aussi le chômage de masse et l'aggravation des conditions de travail qui orienteront les syndicats vers de nouvelles questions sociales, au détriment de celle du pouvoir d'achat, et donc de l'indice des prix et de sa mesure.

\subsection{De l'indexation des salaires à celle des produits financiers}

Le rôle joué par l'indexation des salaires et des prestations sociales sur la sensibilité politique vis-à-vis de l'indice des prix déplace une partie des luttes et controverses dans le champ de la statistique, ce que l'épisode CGT montre clairement. Une innovation de l'État financiarisé, l'indexation des produits financiers à la fin des années 1990, vient, ultérieurement, accentuer cette fébrilité. Mobiliser l'indice des prix pour indexer certains produits financiers donne à voir comment l'État «se remet à vivre dans la dépendance du capital [...] appelle et met en place un capital financier qui va vivre de l'État en se nourrissant notamment de sa dette publique » [Théret, 2010, p. 141]. L'indexation des produits financiers depuis les années 1990 constitue un des dispositifs d'équipement du marché financier, et rassure les prêteurs [Tinel, 2016, p. 50]. Depuis 1997 le dispositif du Treasury Inflation Protected Securities (TIPS) est mis en place pour fournir un retour sur investissement ajusté de l'inflation [Greenlees et McClelland, 2008, p. 4].

Un an après les États-Unis, le Trésor public français émet autour de $10 \%$ de sa dette avec des obligations indexées sur l'indice des prix (OATi ou OATie). Ce dispositif émane du ministre des Finances de l'époque, Dominique Strauss-Kahn [Lemoine, 2016, p. 145], et indexe, en quelque sorte, le taux d'intérêt sur le taux d'inflation pour protéger les créanciers des risques inflationnistes: ces créanciers (par exemple des fonds de pension) paient souvent par ailleurs des flux indexés sur l'inflation (retraites, rentes, livrets réglementés, etc.). Ils sont ainsi protégés de ces risques inflationnistes par ces OATi, qu'ils achètent certes souvent à des rémunérations inférieures aux taux fixes, mais ajustées des taux d'inflation, ce qui leur permet de récupérer leur mise.

Un rapport très complet de Moati et Rochefort en 2008 revient sur une partie des disputes qui traversent la mesure de l'indice des prix. Ce rapport suggère la production d'une pluralité d'indices de prix, selon les usages. Les banquiers interviennent dans le débat en se positionnant en soutien de la politique de l'indice unique de 
I'Insee. Commentateur du rapport Moati et Rochefort, le banquier de BNP-Paribas, Delpla, insiste ainsi sur le rôle déterminant des DSK bonds comme consolidateur de la financiarisation. II estime au total à 150 milliards d'euros le montant de la dette publique indexée sur I'IPC et conclut : "Si les critiques actuelles devaient continuer et mettre en question l'intégrité de I'IPC, si les investisseurs venaient à croire que I'IPC sous-estime sérieusement l'inflation en France, alors il y a un risque sérieux qu'ils vendent leurs OATi ou qu'ils demandent, pour rester investis en OATi ou en CADESi, une indexation sur un indice montrant beaucoup plus d'inflation. Ce serait un coup terrible pour les finances publiques, pour la crédibilité financière de la France et pour le programme d'endettement en dette indexée sur les prix. Jusqu'ici le débat sur I'IPC n'a pas été considéré comme sérieux par les investisseurs. Mais si ce devait être le cas, comment le Trésor et la CADES continueraient leur programme de financement des OATi et des CADESi ? » Et de conclure : "C'est pour cela que le gouvernement, et notamment le ministère des Finances, devrait faire en sorte de mettre un terme rapide à cette polémique sur I'IPC français et ne pas créer plusieurs indices globaux des prix, sinon il y a risque d'écorner dix ans de crédibilité du Trésor sur les marchés obligataires» [Delpla, 2008, p. 166]. Les nouvelles indexations de la fin des années 1990 concernent donc moins les salariés que les créanciers, et les banquiers s'érigent en acteurs du débat.

\subsection{Les années « ante » et « post-Boskin » aux États-Unis, puis en France}

II n'est pas inintéressant de se pencher sur le cas américain des années 1950, car la période conditionnera les idées à venir, y compris en France. La forte progression de l'inflation enregistrée dans les années 1950 aux États-Unis, ainsi que les politiques d'indexation qui s'en sont suivies, sont gérées très différemment de la France. Deux économistes, Ruggles de l'Université de Yale, et Rees de l'Université de Chicago, dans un Joint Economic Committee en 1958 présidé par George Stigler ${ }^{9}$, innovent radicalement dans le choix des arguments éclairant le regain d'inflation américaine. Plutôt que d'insister sur le rôle des syndicats ou sur celui des monopoles industriels dans la progression de l'inflation, Ruggles et Rees font valoir un nouvel argument. La statistique publique serait, selon eux, dans l'incapacité de « capter une forme centrale de la croissance des économies post-guerre : l'innovation telle qu'elle est reflétée dans la qualité des produits et les produits nouveaux » [Ruggles et Rees, 1959]. Cette commission marquera les premières interrogations sur la difficulté de la mesure statistique des prix à capter les effets « qualité » [Stapleford, 2009]. Elle produira aussi les premières recommandations d'économistes en vue d'améliorer la méthode de mesure des indices de prix, visant à mieux tenir compte de l'introduction de nouveaux produits et à mieux refléter les variations de qualité et de productivité. Sur la suggestion de Stigler, la notion d'analyse à « utilité constante des indices du coût de la vie » sera introduite dans le manuel de la statistique publique américaine. Dès 1959, Stigler préconise déjà en effet que I'IPC devienne un indice « à utilité constante » ou un « indice de bien-être », suggérant parallèlement qu'un tel indice aurait enregistré

${ }^{9}$ École de Chicago, prix de la Banque de Suède en 1982. 
de plus faibles augmentations des prix depuis la Deuxième Guerre mondiale que ce que ne laisse pas entendre la mesure officielle des prix [Stapleford, 2009, p. 313].

Ces recommandations seront assez peu suivies d'effets et il faudra attendre 20 ans, pour que la question resurgisse, au moins dans le débat public américain. En 1995, à la demande d'Alan Greespan, président de la Fed, une commission présidée par Michael Boskin sur la mesure de l'inflation connaît un certain retentissement médiatique, tant au niveau américain qu'au niveau international. Cette commission reprend à son compte les principaux arguments portés dans les années 1950 par Ruggle et Rees. Mais alors que les résultats précédents étaient restés relativement confidentiels, on doit cette fois la grande médiatisation du rapport Boskin à la quantification des écarts entre la mesure de l'indice des prix officiel et celle qui devrait être réalisée - selon les économistes - pour la rapprocher d'un « indice du coût de la vie ». C'est cette quantification qui circule largement dans les médias, la Commission de Boskin et al. mettant en avant une surestimation assez spectaculaire de l'inflation d'environ 1,1\% par an en 1996, et de 1,3\% par an pour les dix années antérieures.

Les grandes sources de biais identifiées par le rapport sont liées aux stratégies de substitutions des consommateurs dans l'achat de biens dont I'IPC ne rendrait pas compte, à l'introduction de plus en plus fréquente de nouveaux biens dans la consommation, et à une sous-estimation supposée de la qualité de ce qui est consommé. Thomas Stapleford avance la thèse que le projet théorético-politique en marche depuis les années 1950 vise de facto à soutenir scientifiquement que l'inflation serait surestimée. C'est en tout cas le projet et la conclusion à laquelle aboutit la commission Boskin. Boskin propose des innovations dans la mesure de l'indice des prix qui, toutes, ont vocation à atténuer cette supposée surestimation. Le contexte politique et économique est par ailleurs favorable à de telles réformes dans le calcul de l'indice : outre que la période est marquée par la fin de la guerre froide, le déficit structurel de la balance américaine des paiements et la contestation de plus en plus forte du dollar comme monnaie de référence [Théret, 2010, p. 149] sont autant de facteurs qui incitent à débusquer des sources de réduction des déficits. II est frappant de lire, en introduction du rapport de Boskin et al., une insistance sur l'aggravation du déficit fédéral américain : « Le Congrès et le Président doivent décider s'ils souhaitent continuer la surindexation substantielle et étendue de différents programmes de dépenses fédérales et de différents critères du code des impôts » [p. 3]. Selon Stapleford, le gouvernement américain trouve, par une alliance avec ces économistes standards plaidant pour une réforme de l'indice, un véritable levier d'action pour baisser les dépenses publiques.

En France, mais cette fois en 2008, des économistes s'inquiètent, sur un registre tout à fait comparable, à des propositions pourtant stimulantes de Philippe Moati et Robert Rochefort [2008] visant l'établissement de plusieurs indices des prix. Ainsi, le banquier Delpla, sollicité pour réagir à ce rapport produit pour le compte du conseil d'analyse économique dit : "Si I'Insee publiait plusieurs indices de prix, montrant une plus forte inflation, les demandes de revalorisation des prestations sociales et des minima sociaux aggraveraient un peu plus les déficits publics »[Delpla, 2008, p. 166]. 
Au niveau mondial, ce ne sont plus les syndicats, mais des expertises alternatives émanant d'économistes critiques [Baker, 1998] ou de blogs alternatifs qui s'emparent cette fois de la discussion de la mesure des prix. Les contributions écrites de Williams, sur le site Shadowstat font d'autant plus parler d'elles aux États-Unis que l'auteur fournit des graphiques saisissants suggérant une nette réduction de la tendance de l'inflation depuis les premières réformes méthodologiques de I'IPC aux ÉtatsUnis, par rapport à la tendance qui aurait été enregistrée sans ces modifications. Ces réformes méthodologiques (passage à un Laspeyre chaîné, recours à des moyennes géométriques pour les micro-indices, décote sur l'augmentation des prix pour tenir compte de l'évolution de la qualité) sont considérées par certains auteurs comme de véritables dispositifs « dévaluatifs ». Ces analyses alternatives connaissent aux ÉtatsUnis une couverture médiatique suffisamment étendue pour que s'arme une contreattaque des statisticiens du Bureau of Labor Statistics (BLS), dont le directeur de la division des prix lui-même. Dans un long article du Monthly Labor Review, Greenlees et McClelland [2008] critiquent vertement l'idée que l'inflation puisse être, par les différentes réformes qu'ont connues les États-Unis depuis la première conception de I'IPC, sous-estimée. Ils accordent même à une partie de ces estimations alternatives et des critiques « répétées par de nombreux blogueurs et commentateurs » qui les accompagnent, le statut de "légendes urbaines » [op. cit., p. 6]. Ils espèrent ainsi éteindre l'incendie de la sérieuse dispute, ou tout au moins décrédibiliser leurs pourfendeurs. La défense des statisticiens du BLS prend plusieurs formes: sont avancés des arguments sur le large « consensus scientifique » autour des théories du coût de la vie et de l'utilité constante, des arguments sur la « consistance » avec «les standards internationaux » et les "manuels statistiques», ou encore sur la solidité et la rigueur des méthodes économétriques des prix hédoniques. Des arguments d'autorité sont ajoutés, mettant en avant, pour défendre leur argumentaire, le statut de « prix Nobel » de Stigler.

Là encore, 20 ans plus tard, mais cette fois en Grande-Bretagne, un rapport intitulé le «Johnson Review» est parfois considéré comme le "Boskin britannique». Ce rapport britannique vise à fournir les arguments du passage d'un indice des prix à panier fixe de type Laspeyres, à un indice du coût de la vie [O'Neill et al., 2017, p. 266]. En France, et c'est sans doute un paradoxe, la controverse est importée à la suite de la commission Boskin de 1996 par des chercheurs plutôt « progressistes » qui portent d'abord un intérêt à ce que les indicateurs disent d'une autre représentation des richesses [Gadrey, 1996, 1999]. Gadrey insiste alors plutôt sur les propositions assez radicales auxquelles le rapport Boskin aboutit pour régler le supposé problème de surestimation de l'inflation. II souligne entre autres le caractère innovant des propositions en matière de mesures des outputs et des résultats dans des activités pour lesquelles l'idée même d' « unité de production » n'a pas de sens (notamment dans la santé ou l'éducation), rendant délicate toute mesure de l'évolution des prix (voir encadré 1).

La controverse suscitée outre-Atlantique par le rapport de la commission Boskin en 1996 fait peu de vagues en France. II faut dire qu'elle est largement contenue par les statisticiens de l'Insee, qui s'inquiètent des répercussions possibles de ce rapport visà-vis de la confiance dans l'indice officiel de l'Insee. L'Institut réagit très rapidement 
pour couper court aux controverses qui pourraient circuler, par la publication de deux articles simultanément. L'un, de François Lequiller dans Économie et Statistique, et l'autre de Michel Glaude, alors directeur des statistiques démographiques et sociales de I'Insee, dans La Revue française du marketing. Glaude n'hésite pas à considérer que "le débat américain semble avoir dérapé », certaines critiques relevant " de l'opportunisme politique », et de l'« excès d'habileté d'Alan Greenspan pour justifier une politique monétaire qui se contente, de fait, d'un glissement annuel des prix de détail de 2 à $3 \%$ » [Glaude, 1997, p. 20]. Quant à Lequiller, à l'époque « chef adjoint du département des prix à la consommation, des ressources et des conditions de vie de l'Insee, chargé de l'indice des prix à la consommation français », il vient de passer plusieurs années aux États-Unis, auprès du FMl, qui le rendirent alerte sur les controverses occupant les Américains. II rédige une contribution particulièrement fouillée. Son article, auquel chercheurs comme syndicats continuent de se référer 20 ans plus tard, considère que la France est plutôt à l'abri d'une telle surestimation, que Boskin aurait dû se dispenser de proposer une évaluation chiffrée du « biais » : « Avancer un chiffre quelconque sur ce problème dans l'état actuel de nos connaissances n'est guère raisonnable » [Lequiller, 1997, p. 16], insistant même : "Dans un cas comme celui-là, la réponse du statisticien doit être de reconnaître son ignorance et de travailler à la réduire et non à faire des estimations hasardeuses » [ibid., p. 18]. Lequiller admet, par ailleurs, que l'essentiel de l'estimation du rapport Boskin repose sur le problème de la substitution des biens, mais que, sur un certain nombre d'autres aspects (qualité des produits, estimation des activités de services, tension entre utilité constante et panier de biens constants, etc.), des améliorations sont encore envisageables, sans présager du signe +/- par lequel l'indice pourrait être affecté. Il entrevoit néanmoins trois voies d'amélioration de l'indice - qui constitueront trois réformes discrètes ultérieures de l'indice, aux destins plus ou moins heureux : d'abord une accélération du recours aux méthodes économétriques (hédonistes) « [au-delà du calcul sur les micro-ordinateurs], cette voie continue d'être explorée et une méthode économétrique d'estimation des effets qualité sera bientôt opérationnelle pour certains autres biens durables (lave-vaisselle notamment) », un approfondissement des synergies entre instituts européens de la statistique, et, enfin, l'usage plus massif des données en provenance des sociétés privées d'études de marché qui «figureront probablement parmi les solutions d'avenir pour la correction des biais de substitution de niveau détaillé et de nouveaux circuits de distribution » [ibid., p. 24].

Si, par son article, Lequiller vise ainsi à apaiser une controverse naissante, et s'il considère que I'IPC $a$, en France, un degré de fiabilité élevé, d'autres économistes réactivent les questions quelques années plus tard, avec nettement moins de nuances, et concluent à une probable surestimation de l'inflation en France. À la suite de Da Costa [2001] et Hamilton [2001] pour les États-Unis, puis de Beatty et Larsen [2005] pour le Canada, Clerc et Coudin [2010] appliquent une méthodologie dite « de la courbe de Engel », qui se diffuse rapidement au-delà du continent américain. Quantifiant la différence entre I'IPC et un indice du coût de la vie, les statisticiennes concluent, à partir de travaux économétriques réalisés sur l'enquête Budget des familles, que l'inflation en France aurait surestimé le coût de la vie de $3 \%$ par an entre 1974 et 1981, puis de $1 \%$ par an de 1981 à 1994. Ces travaux, qui affirment que l'inflation serait surestimée, visent cependant moins à se placer dans le débat Boskin-Lequiller, que dans 
la nouvelle dispute qui commence à poindre depuis la construction de la zone euro (traité de Maastricht, puis l'introduction de l'euro).

\section{Encadré 1. Le rapport Boskin et les mesures du bien-être}

Le rapport Boskin [1996], remis à la commission des finances du Sénat américain en 1996, porte d'abord sur les biais de la mesure de l'inflation, celle-ci étant jugée comme surestimée. Le biais de la mesure de l'inflation est même considéré comme « le quatrième poste des dépenses fédérales après la sécurité sociale, les dépenses de santé et la défense » (p. 2). Ce faisant, Boskin établit que le PIB en volume et la croissance seraient de leur côté sous-estimés, rejoignant les thèses d'Aghion [2017], soutenues 20 ans plus tard.

Mais en faisant des suggestions sur les mesures de volume de certains services, et de leurs impacts, Boskin va au-delà de cette discussion comptable et sa représentation de la réalité surprend. Alors qu'il défend l'idée d'un indice du coût de la vie (Coli $\left.{ }^{10}\right)$, Boskin affirme aussi "dans l'absolu, pour l'obtention d'un Coli, il ne faut pas se limiter aux changements de comportement des consommateurs. II faut aussi tenir compte des effets négatifs des taux de criminalité (conduisant à l'achat d'équipement de sécurité, ou encore d'assurances) et des effets négatifs liés par exemple au télétravail permettant à un parent d'en profiter pour garder son enfant » (p. 21). La manière de traiter ce qu'il appelle les externalités, comme les hivers rigoureux, l'apparition du sida (on est au début des années 1990) ou encore l'augmentation du taux de criminalité, la multiplication des divorces, n'est pas précisée, mais il invite à approfondir dans le futur les recherches sur ces questions. Pour ce qui est des résultats de ce rapport, dit-il, les « bons » éléments contrebalancent cependant les « mauvais », et la société progresse. Ce serait, selon lui, dans le domaine de l'environnement et de la santé, que les choses auraient nettement progressé aux États-Unis. S'ensuit un paragraphe indiquant, de manière quasi lyrique, à quel point la société américaine a connu des progrès en matière écologique par rapport aux années 1950 et 1960 (par ex. : «the coal scuttle was replaced by the silent and automatic transmission of natural gas » ou encore : « the rapid spread of central air conditioning has made possible a substantial movement of households to the southern and south-western states »). Au total, le visionnaire Boskin note que les pollutions, crime, suicide, divorce " ont atteint un plateau » (p. 49) et, «dans le cas de la pollution et du crime, la tendance semble s'être inversée, améliorant donc depuis récemment la qualité de vie ».

\section{Le traité de Maastricht, le pouvoir croissant d'Eurostat et le passage à l'euro}

Le traité de Maastricht de 1992, qui établit I'Union économique et monétaire (UEM) des pays de la zone euro, fixe des critères de convergence que ces pays doivent respecter avant et après l'intégration, en particulier un déficit public inférieur à $3 \%$ du

\footnotetext{
10 "Cost of life index»; opposé au "Cogi», " cost of goods index» qui s'intéresse aux dépenses nécessaires pour l'achat de la même structure et quantité de biens.
} 
PIB et une dette publique inférieure à $60 \%$ du PIB. En matière de taux d'intérêt et d'inflation, le traité stipule que les taux ne doivent pas dépasser ceux des pays les moins inflationnistes. Les pays visant l'adhésion au traité préparent donc, dès la fin des années 1980, à la fois la mise en place de politiques rigoureuses visant le respect des critères européens [Boyer, 2015, p. 174], et une plus grande coordination dans les méthodes de mesure de l'inflation. Cette coordination devient même un impératif $\mathrm{d}^{\prime}$ Eurostat. Difficile à assurer alors que les pays disposent de systèmes statistiques inégaux, Eurostat produit dès 1996 un Indice des prix à la consommation harmonisé (IPCH), fixé par règlement et construit à partir des données de prix collectées par les pays. Eurostat s'installe comme institution internationale décisive, par une succession de règlements et directives ayant force de loi, dans l'harmonisation des définitions, des nomenclatures, des méthodes et couverture de mesure des prix dans les pays de I'UEM. L'IPCH est retenu par la BCE comme indicateur de stabilité des prix et ces dispositifs préparent le passage à l'euro.

\section{Graphique 1. Évolution de l'écart entre perception et mesure officielle de I'inflation. France : 1970-2016}

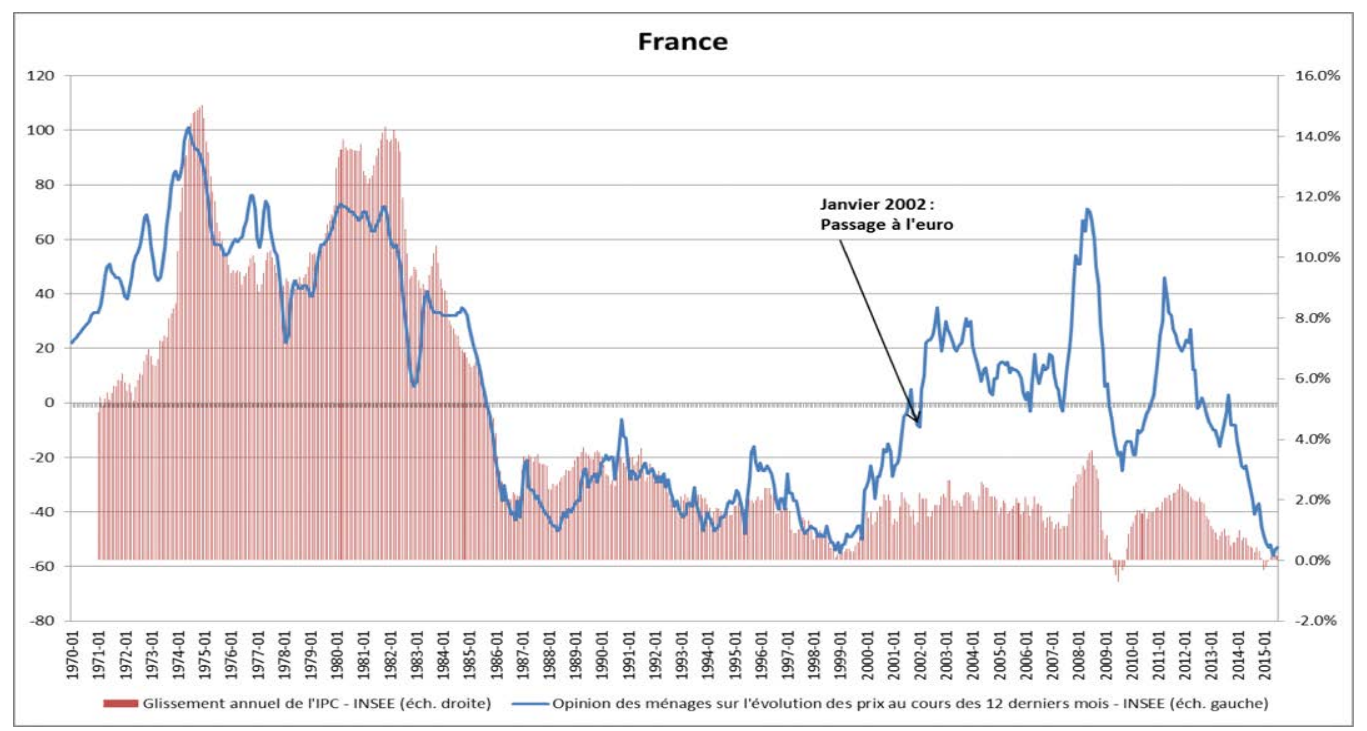

Source: Pionnier, 2015.

\subsection{Un hiatus persistant entre la perception des ménages et l'indice officiel}

L'arrivée de l'euro en 2002 produit divers effets, parmi lesquels un effritement de la confiance des ménages à l'égard de la monnaie. Effritement qui se traduit par une mise en doute des principaux indices collectifs de l'évolution des prix dont les médias rendent compte tout au long de l'année 2002. L'affaiblissement de la crédibilité de l'indice officiel, qui n'est pas propre à la France, conduit d'abord à la mise en place dès 2004 d'une refonte de l'enquête de conjoncture auprès des ménages (Camme). Un module "conjoncture», composé des questions harmonisées au niveau européen, et comprenant une partie « opinion sur la conjoncture générale en France », sur les 12 mois passés et sur les 12 mois à venir (situation économique 
générale, chômage, inflation, opportunité d'acheter, d'épargner...) » [CNIS, 2013], recueille la perception des individus sur l'inflation ressentie. Cette enquête permet de souligner un hiatus net entre inflation "perçue » et inflation de la statistique publique, partout en Europe (voir graphique 1). Un rapport commandité par la BCE est réalisé par des économistes [Dieden et al., 2006]. II fait état d'écarts très nets dans tous les pays européens entre perceptions et indice officiel, y compris en France. II est estimé que l'écart est en permanence de l'ordre de six points entre 2004 et 2010 [Accardo et al., 2011, p. 4].

Le débat trouve un nouvel ambassadeur, par le biais de Leclerc, directeur du réseau d'hypermarchés éponyme, qui se fait l'officiel défenseur du pouvoir d'achat des ménages, promouvant ainsi sa politique de prix bas et se substituant à des syndicats en retrait. En février 2004, à travers une campagne publicitaire explicite, le distributeur conteste le diagnostic de l'Insee de 2003 sur le pouvoir d'achat des ménages. Le ministre de l'Économie et des Finances de l'époque (Sarkozy) joue un rôle décisif dans la montée de cette polémique, dénonçant régulièrement les hausses de prix dans la grande distribution. Leclerc exige qu'un indice des prix puisse rendre compte de l'évolution des prix transitant par ce type de point de vente ; injonction à laquelle le gouvernement accède - obligeant aussi I'Insee à s'exécuter - au grand dam de nombreux syndicats et professionnels de l'Institut de la statistique.

\subsection{Les différents niveaux de réponse de l'Institut de la statistique}

Les instituts de la statistique répondent à cette perte de confiance dans la mesure au moyen de divers outils. D'abord, par une mise en visibilité plus nette des indices de perception de l'inflation venant objectiver les écarts entre inflation « réelle » et «perçue ». Le directeur de l'Insee de l'époque reconnaît que ces écarts sont historiquement élevés [Data, 2009, p. 28], ce que les travaux d'Accardo [Accardo et al., 2008] ou de Pionnier [2015] illustrent également (voir graphique 1).

Au débat sur le "bon» niveau de l'inflation que connaissent les pays européens, divers facteurs explicatifs sont mis en avant. Est d'abord avancée l'idée d'une hétérogénéité croissante des populations sur laquelle l'Insee se penche un temps : la perception de l'inflation ne serait pas vécue de la même manière selon les catégories sociales. Dans un long dossier, «La mesure du pouvoir d'achat et de sa perception par les ménages » [Accardo et al., 2008], I'Insee propose des indices de prix catégoriels, c'est-à-dire différents selon les classes sociales. Les résultats sont décevants, de l'avis même de l'Insee, car ils montrent, contrairement aux intuitions, une très faible influence de la catégorie sociale sur les variations de l'indice des prix. Une des raisons tient au fait que ces «indices de prix catégoriels » ne peuvent pas, en l'état de la collecte, tenir compte d'effets de gamme à l'intérieur d'une variété de produits (les classes sociales ne consomment ni les mêmes « pommes » ni les mêmes « voitures »). Ils reposent seulement sur une déformation des coefficients budgétaires selon les catégories sociales (un poids par exemple plus élevé dans la consommation de produits alimentaires). 


\section{Graphique 2. Simulateur de l'Insee, exemple fictif ${ }^{11}$}

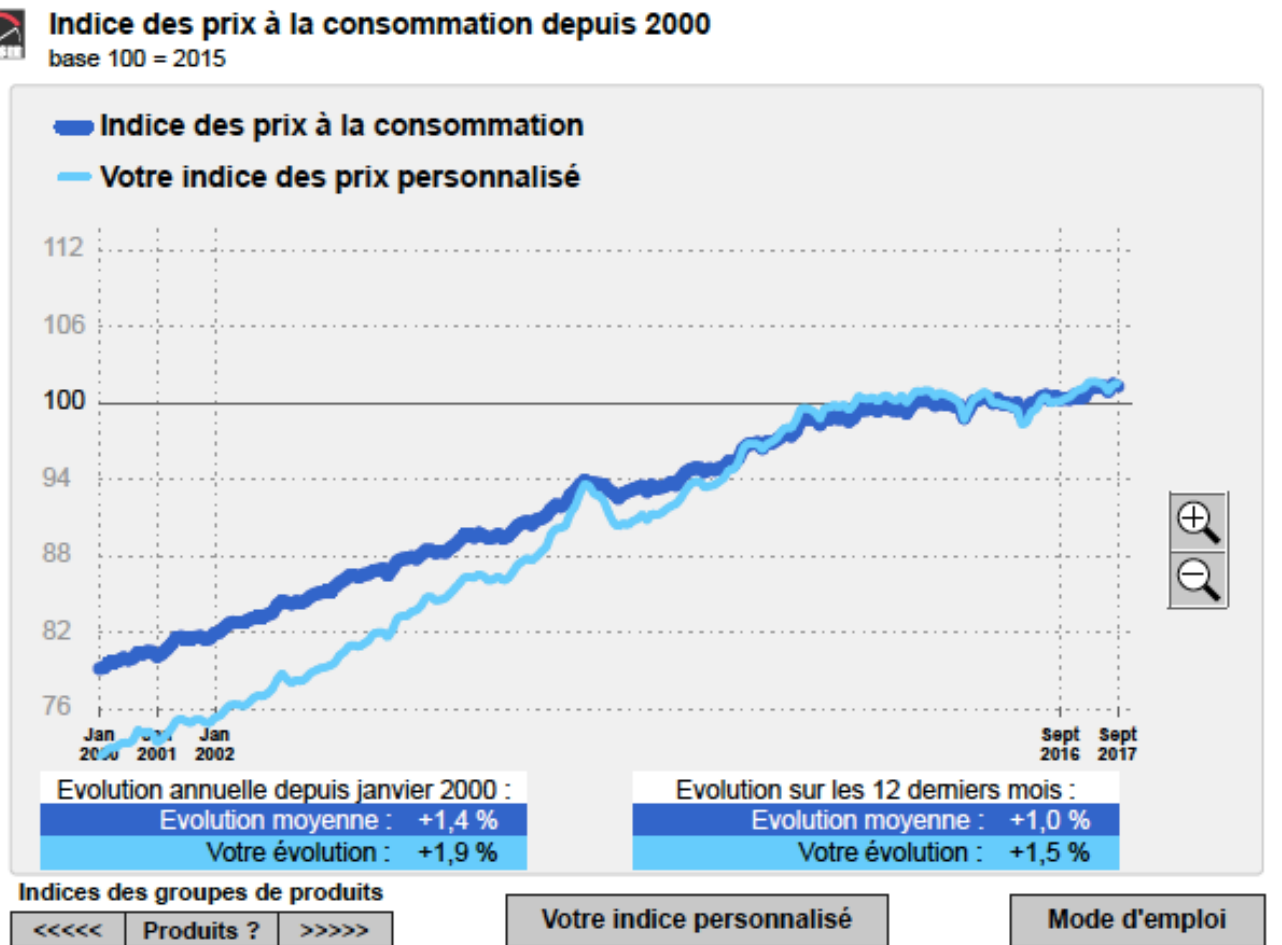

Source: Test personnel sur un IPC «personnel » et l'IPC officiel. Dans cet exemple, l'inflation de l'individu fictif (qui n'a pas de voiture, et consomme peu de biens semi-durables de loisirs) a été de $+1,9 \%$ par an depuis 2000 contre $+1,4 \%$ par an pour l'inflation officielle.

Les instituts nationaux substituent alors dès le milieu des années 2000 à cette analyse des classes un dispositif jugé innovant: un "calculateur individuel». Chaque consommateur a accès en libre-service à un simulateur qui permet, assez instantanément, de mesurer son inflation personnelle... C'est sur la base d'une supposée hyperrationalité calculatoire de l'individu que ce dispositif est mis en place. Dès 2006, le site officiel de l'Institut statistique allemand (Destatis) émet une première version d'un calculateur individuel, que chacun peut faire varier selon la structure de sa consommation personnelle. L'initiative est relayée par la revue Sigma. The Bulletin of European Statistics [Neutze, 2006]. D'autres offices statistiques nationaux en Europe lui emboîtent le pas, ce qui suggère à quel point les méthodes statistiques innovantes et les savoirs qui les accompagnent circulent rapidement dans l'espace européen. En Grande-Bretagne dès janvier 2007, deux statisticiens de l'Office for National Statistics décrivent comment I'Institut statistique national britannique introduit un site dédié au calcul de l'inflation individuelle... En France, durant le premier trimestre 2007, I'Insee procède d'une même intention en s'inspirant directement de l'expérience allemande. II met en ligne un simulateur (voir graphique 2) à disposition de chaque internaute. La manière de présenter les tendances pourrait d'ailleurs être discutée, la base 100 fixée en fin de période laissant transparaître, ce qui n'est pas exact, l'idée d'un certain « rattrapage » des inflations personnelles par rapport à l'inflation officielle.

${ }^{11}$ Téléchargement le 9 octobre 2017. 
Les instituts de la statistique visent par ce simulateur personnel plusieurs objectifs : contenter le consommateur individuel, apaiser les controverses collectives, aider à la reconstruction d'une légitimité de l'indice, mais aussi faire gagner en légitimité l'idée qu'un indice du coût de la vie serait plus adéquat. En gagnant en pertinence individuelle, ces instituts de la statistique publique pensent ainsi que la légitimité autour de la production de l'indice moyen pourra être sauvegardée. Cette nouvelle offre statistique est aussi l'expression de la prévalence d'une logique d'État qui associe à son mode de gouvernance une démultiplication des centres de calcul et une individualisation croissante des dispositifs évaluatifs [Desrosières, 2008]. L'indice des prix calculé par chacun devient ici un mode de description statistique personnalisé, qui, sans avoir de statut légal, permet à chaque « agent » de se mettre en "ordre», en adoptant un comportement rationnel et optimal [Jany-Catrice, 2007]. Ces dispositifs promeuvent une conception très appauvrie de la statistique publique et font perdre aussi, ce faisant, aux instituts de la statistique publique leur ligne d'horizon de production de dispositifs cognitifs collectifs de coordination des acteurs.

\subsection{La maîtrise des dépenses publiques et l'émergence des données de caisse}

L'entreprise de réhabilitation de la confiance dans l'indice des prix de l'Insee passe également par de nouveaux investissements, car l'écart entre indice perçu et indice réel reste très marqué en France jusque 2016, contrairement aux autres pays européens. Des négociations entre 2010 et 2016 avec la grande distribution conduisent à la mise en place d'un dispositif innovant de « données de caisse ». L'idée est déjà présente dans le rapport Boskin, mais peu abordée dans la littérature française jusqu'au début des années 2000, même si Eurostat met le dispositif à son agenda dès la fin des années 2000. L'accès direct aux données de caisse de la grande distribution ainsi permis grâce à la Loi numérique de 2016 est motivé par une nécessité de baisse des dépenses de l'Insee. Les 200000 relevés mensuels réalisés dans les différents points de vente par les enquêteurs (« collecte terrain »), auxquels s'ajoutent 190000 collectes de prix (par voie d'internet, courrier, ou voie administrative), ont, en effet, un coût important en période de RGPP et de «maîtrise » des dépenses publiques. L'Insee suggère que « les moyens utilisés sont de 20 personnes à la division des prix, une dizaine de personnes au pôle de Bordeaux, une soixantaine d'agents en sites "prix" (métropole et Dom) et 200 enquêteurs repartis sur les sept sites de métropole et les quatre sites des Dom. Une équipe de maintenance informatique de 6 personnes est en charge du développement et de la maintenance des applications » (CNIS, 2018).

II s'agit aussi pour l'Insee de faire figure symbolique d'innovateur en se positionnant sur l'espace des « big data », de gagner en légitimité, le calcul scientifique et digital des variations de prix permettant d'assurer réputation et légitimité des outputs de l'institution publique. L'Insee met également en avant la possibilité, par le biais de ces données, d'avoir accès pas seulement aux prix mais aussi aux quantités achetées, de résoudre une partie des problèmes d'évaluation de la substitution des biens, au niveau des "micro-indices», voire, suggèrent des travaux américains, de résoudre une partie des problèmes d'évaluation de la qualité [Silver et Heravi, 1999]. 
Une partie du traitement des données peut être considérée comme de la responsabilité de nouveaux acteurs qui entrent dans le jeu de la production de l'IPC: des " concentrateurs », en effet, sont amenés à jouer un rôle important dans la construction de l'indice. II s'agit d'agences marketing leaders sur le marché de la grande distribution (ACNielsen et IRI). Ils deviennent une interface décisive entre I'Insee et les flux de sortie des données de caisse. Afin d'obtenir les spécifications techniques qui sont retenues pour déterminer la qualité des produits et leur équivalence (au-delà des codes-barres), des catalogues produits sont réalisés et utilisés par ces agences. La nomenclature des codes-barres, indispensable pour tenter d'apprécier les « glissements de qualité », est collectée et définie par ces organismes privés, ce qui transmet de facto le "pouvoir de valorisation» de la qualité [Eymard-Duvernay, 2012; Bessy et Chauvin, 2013], jusqu'ici contrôlé par une autorité publique, à ces acteurs privés, avec tout ce que ce pouvoir de valorisation intègre comme dimensions cognitive, éthique, sociale et politique. Ces intermédiaires qui ont, les premiers, au niveau mondial, contacté les offices nationaux de la statistique pour leur vendre leurs données dès la publication du rapport Baskin, n'ont pas seulement pour rôle de faciliter les flux et transferts d'informations. Ils contribuent pleinement à la définition des conventions de qualité des produits (biens et services) qui entrent dans la composition des prix.

L'idée de ce dispositif est introduite en France, de manière concomitante avec l'idée d'indice à utilité constante, d'abord chez Magnien et Pougnard [2000], puis chez Sillard [2013], dans la lignée des travaux de Stigler. L'indice à utilité constante est la définition contemporaine de l'indice du coût de la vie. L'indice du coût de la vie, ou Coli, mesure, selon la théorie, la variation des dépenses nécessaires pour l'obtention d'un même niveau d'utilité, laissant d'ailleurs entendre que le prix, et la formation de l'inflation, seraient directement issus de l'utilité subjective individuelle. Alors que, dans un indice de Laspeyres, les quantités sont exogènes, dans le Coli, les quantités sont expliquées par le comportement rationnel des consommateurs [Breuer et von der Lippe, 2011]. L'indice à utilité constante entre donc en pleine cohérence avec la théorie standard, mettant ainsi la statistique publique au service de la théorie économique : le projet des données de caisse de l'Insee a pour objectif, dit Sillard, "de se rapprocher des fondements microéconomiques des indices de prix à la consommation en réalisant, avec les données de caisses, des calculs d'indices qui tiennent compte de l'éventuelle sensibilité des consommateurs aux prix relatifs des produits. Ces fondements font appel au concept d'indice à utilité constante » [op. cit]. Magnien et Pougnard précisent de manière tout à fait explicite que cette approche repose « toutefois sur une hypothèse fondamentale : l'existence d'une fonction d'utilité par rapport à laquelle les choix des consommateurs sont rationnels. Cela signifie qu'à chaque période, les quantités acquises maximisent le niveau d'utilité sous la contrainte de budget [...]. On dit qu'une telle fonction d'utilité rationalise les données (prix et quantités) » [Magnien et Pougnard, 2000, p. 82. Ils soulignent].

Sur la question de la qualité, la méthode hédonique - longtemps considérée, en France comme aux États-Unis, comme la méthode la plus prometteuse - fait aujourd'hui place aux nouvelles promesses de ces concepts d'indice à «utilité 
constante », (ré)habilitant ainsi les indices du coût de la vie, empruntant des voies théoriques pleinement utilitaristes, et engageant de nouveaux choix méthodologiques. Ce projet, qui sera testé en format réel en 2019 par l'Insee et mis en place en 2020, peut aussi être interprété comme un grand renversement : «En faisant de l'État non plus la chose mesurante et planificatrice de l'économie [...], mais bien la chose mesurée par les acteurs privés de l'économie » [Gayon et Lemoine 2014, p. 31].

\section{Conclusion}

Loin des subordinations directes à un gouvernement singulier qui pouvait caractériser les années 1950, la statistique publique est en proie aujourd'hui à des interdépendances, plus discrètes peut-être, avec le politique. Le cas de la mesure de l'inflation le montre : les disputes mettent en scène de nouveaux acteurs et tendent à les « confiner » entre experts économistes et politiques. Cette migration des disputes est liée aux citadelles techniques (technicité des méthodes, technicité de la sémantique) qui sont érigées entre la société civile et l'outil statistique, qui fournissent parfois une illusoire aura apolitique aux économistes, mais liée aussi, il faut le reconnaître, à une fragilisation syndicale et à son retrait de débats pourtant importants.

L'Insee est confronté à la recherche de solutions, tout en ayant le souci permanent de garantir la solidité de l'institution via la stabilité de ses conventions statistiques et comptables. Ainsi, pour le moment, le projet des données de caisse se fait toujours sur un indice de Laspeyres, même si ce raccord méthodologique pose de redoutables questions méthodologiques. De son côté, la théorie économique standard approfondit ses recherches et attend le «moment opportun» [Vatin, 2013] pour assurer, par l'entremise de la statistique publique, une mise en pratique. Les innovations concrètes qui font glisser le «vieil » indice des prix vers un indice du coût de la vie (chaînage, moyennes géométriques, méthodes hédoniques, données de caisse, etc.) ont un coût qu'il faut consentir par rapport à la complexification des pratiques économiques. Dans les faits, cette complexification n'est finalement jamais rattrapée, et se « dérobe » sous les outils [Jany-Catrice, 2017, 2019].

Les innovations fournissent ainsi des fondements microéconomiques à cet indice d'ordre macroéconomique. Ces fondements instillent l'idée d'un possible ordre collectif qui ne procéderait pas d'un accord collectif explicite, notamment sur l'épineuse question de la qualité. L'autonomisation de la science économique passe aussi par ces discrets choix-là. 


\section{Bibliographie}

Accardo J. (2005), « L'estimation des échelles d'équivalence dans les enquêtes “Budget de famille": les limites de l'exercice », séminaire DSDS Insee, 18 mars.

Accardo J., Chevalier P., Forgeot G., FriezA., Guédès D., Lenglart F., Passeron V. (2008), « La mesure du pouvoir d'achat et de sa perception par les ménages », Insee: I'Économie française, 2007, p. 58-86.

Accardo J., Célérier C., Herpin N. (2011), "L'inflation perçue », Économie et Statistique, $n^{\circ} 447$, p. 4-31.

Aghion Ph., Bergeaud A., Boppart T., Klenow P.J., Li H. (2017), «Missing Growth from Creative Destruction », Federal reserve Bank of San Francisco Working Paper Series, January 2017 (working paper 2017-04).

Armatte M. (2010), La science économique comme ingénierie. Quantification et modélisation, Presses des Mines, Mines ParisTech, coll. «Sciences sociales», Paris.

Beatty T., Larsen E. (2005), " Using Engel Curves to Estimate Bias in the Canadian CPI as a Cost of Living Index», Canadian Journal of Economics, vol. 38, n² 2, p. 482-499.

BAKer D. (dir.) (1998), Getting Prices Right. The Debate over the Consumer Price Index, Economic Policy Institute, Routledge, London and New York.

Bessy C., Chauvin P.-M. (2013), «The Power of Market Intermediaries: From Information to Valuation Processes », Valuation Studies, vol. 1, n 1, p. 83-117.

Boskin M.J., Dulberger E., Gordon R., Griliches Z., Jorgenson D. (1996), «Toward a More Accurate Measure of the Cost of Living», Final Report to the Senate Finance Committee, Dec. 4.

Boyer R. (1978), « Les salaires en longue période », Économie et Statistique, n 103, septembre, p. 27-57.

Boyer R. (2015), Économie politique des capitalismes, théorie de la régulation et des crises, La Découverte, coll. « Grands Repères », Paris.

BREUER C.C., VON DER LIPPE P. (2011), « Problems of operationalizing the concept of a costof-living index », MPRA, Paper $n^{\circ} 32902$.

CHÉLINI M.-P. (1998), Inflation, État et opinion en France de 1944 à 1952, ed. Comité pour I'histoire économique et financière de la France, coll. «Études générales ».

ClerC M.-E., Coudin E. (2010), «L'IPC, miroir de l'évolution du coût de la vie en France? Ce qu'apporte l'analyse des courbes d'Engel », Direction des études et synthèses économiques. G 2010/04, Insee, document de travail.

CNIS (2018), «IPC. Fiche descriptive pour examen d'opportunité», commission Démographie et questions sociales, 28 novembre.

Court A. (1939), " Hedonic price indexes with automotive examples », in The Dynamics of Automobile Demand, General Motors, New York, p. 98-119.

CoYLe D. (2014), GDP: A Brief But Affectionate History, Princeton University Press, Princeton.

DA Costa L. (2001), «Estimating Real Incomes in the United States from 1988 to 1994: Correcting CPI Bias using Engel Curves", Journal of Political Economy, vol. 109, p. 1288-1310.

$40 \quad{ }_{\text {ser }}^{\text {er }} 2019 \mathbf{R f}_{\text {ge }}^{22}$ 
Data L. (2009), Le grand trucage. Comment le gouvernement manipule les statistiques, La Découverte, Paris.

Delpla J., (2008), «Commentaire», in Ph. Moati et R. Rochefort, Mesurer le pouvoir d'achat, La Documentation française, Paris, p. 163-168.

Desrosières A. (2008), L'argument statistique. Tome 1 : Pour une sociologie historique de la quantification. Tome 2: Gouverner par les nombres, Éd. Mines ParisTech, coll. «Sciences sociales », Paris.

Dieden H., Friz R., Genre V., Le Breton G., Linden S. (2006), « Consumer's quantitative inflation perceptions and expectations in the Euro area: a first evaluation », Report to the European Central Bank, European Commission.

Eymard-Duvernay F. (2012), « Les pouvoirs de valorisation » in B. Roger (dir.) L'entreprise, formes de la propriété et responsabilités sociales, Lethielleux, Paris, p. 176-199.

GADREY J. (1996), Services : la productivité en question, Desclée de Brouwer, Paris.

GADREY J. (1999), «Les incertitudes de l'indice des prix à la consommation: question de méthode ou question de paradigme? » Économie appliquée, vol. 52, n 1, p. 151-175.

Gayon V., Lemoine B. (2014), "Maintenir l'ordre économique. Politiques de désencastrement et de réencastrement de l'économie », Politix, n¹05, p. 7-35.

GLAUDE M. (1997), «Du bon usage des indices de prix à la consommation», Revue française du marketing, $\mathrm{n}^{\circ} 161$, p. 5-20.

Gravel D., (2010), «L'IPC mesure-t-il bien le coût de la vie ? », Économie et Statistique, $n^{\circ} 433-434$, p. 101-105.

Greenlees J., McClelland R. (2008), « Addressing misconceptions about the Consumer Price Index », Monthly Labour Review, August, p. 1-19.

Hamlton B.W. (2001), "Using Engel's Law to Estimate CPI Bias », American Economic Review, vol. 91, p. 619-630.

Hausman J. (2003), "Sources of Bias and Solutions to Bias in the Consumer Price Index », Journal of Economic Perspectives, vol. 17, n 1, p. 23-44.

Jany-Catrice F. (2007), «L'acte de naissance du chacun pour soi », Le Monde, 5 mars.

Jany-CATRICE F. (2017), "Conflicts in the Calculation and Use of the Price Index - the Case of France », Cambridge Journal of Economics, en ligne le 31 octobre 2017. Version papier : 2018, vol. 42, nº 4, p. 963-986.

Jany-Catrice F. (2019), L'indice des prix à la consommation, La Découverte, coll. « Repères », $n^{\circ} 717$, Paris.

LEQUILLer F. (1997), «L'indice des prix à la consommation surestime-t-il l'inflation ? », Économie et Statistique, $n^{\circ}$ 303, mars, p. 3-32.

Lemoine B. (2016), L'ordre de la dette, La Découverte, Paris.

MacKenzie D. (2005), "Opening the black boxes of global finance», Review of International Political Economy, vol. 12, n 4, p. 555-576.

MÉDA D. (1999), Qu'est-ce que la richesse?, rééd. 2008, Au-delà du PIB. Pour une autre mesure de la richesse, Flammarion, coll. « Champs actuels», Paris. 
MoAtı Ph., Rochefort R., (2008), Mesurer le pouvoir d'achat, rapport pour le CAE, La Documentation française, Paris.

Moden J. (1982), "L'indice des prix à la consommation », Courrier hebdomadaire du CRISP, vol. 978-979, n³3, p. 1-38.

Moulton B. (2001), «The Expanding Role of Hedonic Methods in the Official Statistics of the United States», note du Bureau of Economic Analysis U.S. Department of Commerce Washington DC.

Neutze M. (2006), "Calculate your own Inflation Rate», Sigma. The Bulletin of European Statistics, $n^{\circ} 1$.

O'Neill R., Ralph J., Smith P., (2017), Inflation. History and Measurement, Palgrave McMillan, Londres.

OrLÉAN A. (2004), Analyse économique des conventions, PUF, coll. « Quadrige », Paris.

Pionnier P.-A. (2015), «Coût de la vie : perception et réalité statistique », communication Journées d'économie de Lyon, 13 oct.

Powell M., O'Donoghue J. (2007), "The Personal Inflation Calculator», Economic \& Labour Market Review, vol. 1, n 1.

Porter T. (1995), Trust in Numbers: The Pursuit of Objectivity in Science and Public Life, Princeton University Press, Princeton.

ROUCHEt J. (2016), « D'une crise à l'autre. 1907 et la naissance du suivi de la conjoncture à la SGF », colloque des 70 ans de l'Insee, Paris, mai.

Ruggles R., ReEs A. (1959), US Congress, Joint Economic Committee, "Staff Report on Employment Growth, and Price Levels», 86th Congress, 1st sess. December 24, 1959, p. 109.

SAGLIO J. (1993), "Le nouvel indice des prix à la consommation», Économie et Statistique, $n^{\circ} 267$, p. 3-12.

Sillard P. (2013), «Les données de caisse : vers des indices de prix à la consommation à utilité constante », Document de travail Insee n ${ }^{\circ}$ F1305.

SILlaRd P., WILnER L. (2015), «Indices de prix à utilité constante et substitutions intermensuelles », Revue économique, vol. 66, nº 4, p. 755-768.

Silver M., Heravi S. (1999), "The Measures of Quality-Adjusted Price Changes», Proceedings of the Measurement of Inflation Conference, August 31-September 1.

StAPLEFORD T. (2009), The Cost of Living in America. A Political History of Economic Statistics 1880-2000, Cambridge University Press, Cambridge.

THÉRET B. (2010), «L'argent public et les régimes économiques de l'ordre politique. Entretien avec Bruno Théret», Vincent Gayon et Benjamin Lemoine, 2010/3, Genèses, p. 136-152.

Tinel B. (2016), Dette publique: sortir du catastrophisme, Raisons d'agir, Paris.

Tooze A. (2016), «Economic Statistics in the Weimar-era Reichswirtschaftsministerium, the Statistisches Reichsamt and the Institut für Konjunkturforschung », Columbia July, draft. 
Touchelay B. (2014), « Les ordres de la mesure des prix. Luttes politiques, bureaucratiques et sociales autour de l'indice des prix à la consommation (1911-2012)», Politix, n 105 , p. 117-138.

TOUTAIN J.-C. (1996), « Comparaisons entre les différentes évaluations du produit intérieur brut de la France de 1815 à 1938. Ou l'histoire économique quantitative a-telle un sens? », Revue économique, vol. 47, n 3, p. 893-919.

Vanol A. (2002), Une histoire de la comptabilité nationale, La Découverte, coll. « Repères», Paris.

VAtiN F. (2013), «Valuation as Evaluating and Valorizing », Valuation Studies, vol. 1, nº 1, p. 31-50. 